\title{
Automated Reconstruction of Building Facades for Virtual Walk-thrus
}

\author{
Christian Frueh and Avideh Zakhor* \\ Video and Image Processing Lab \\ University of California, Berkeley
}

\begin{abstract}
In this paper, we present a fast, automated approach to generating a highly detailed, textured 3D building facade model. This model is acquired at the ground level by driving a vehicle equipped with laser scanners and a digital camera under normal traffic conditions on public roads, and then processed offline. We evaluate our approach on a large data set acquired in downtown Berkeley.
\end{abstract}

\section{Introduction}

Three-dimensional models of urban environments are useful in a variety of applications such as gaming, urban planning, and virtual heritage conservation. Models reconstructed from airborne data such as aerial images or airborne laser scans generally lack information about the facades or details at the ground level; in addition, they are typically created via a labor-intensive process. Previous attempts to create ground-based models use 3D scanners, requiring a slow stop-and-go mode for the data acquisition, and hence are limited to a few buildings. Recently, we proposed a method that is capable of rapidly acquiring 3D geometry and texture data of an entire city at the ground level [Frueh and Zakhor, 2001a].

\section{Data Acquisition and Model Generation}

Using a combination of two fast 2D laser scanners and digital cameras mounted on a truck, as shown in Figure 1, we acquire data continuously while driving at normal speeds on public roads, rather than in a slow stop-and-go fashion. One 2D scanner is mounted vertically and scans the buildings and street scenery as the truck drives by. The other one is mounted horizontally and is used to determine the vehicle's pose via scan-to-scan matching [Frueh and Zakhor, 2001a], and to globally register the facades with respect to an airborne edge map by means of Monte-Carlo Localization [Frueh and Zakhor, 2001b].
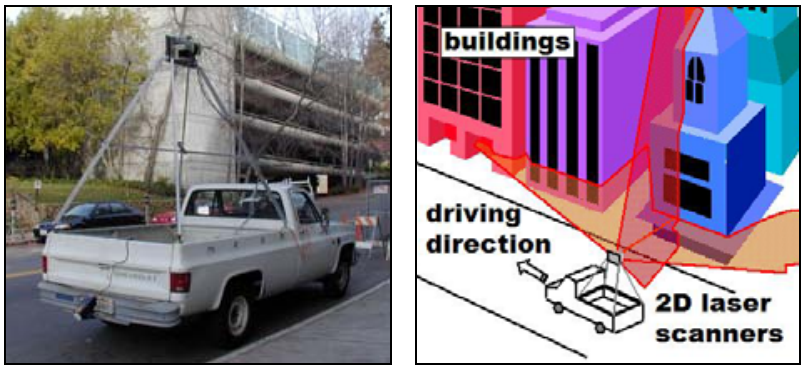

Figure 1. Acquisition vehicle and setup.

After a series of automated processing steps including removal of foreground objects and filling holes [Frueh and Zakhor, 2002], we reconstruct the $3 \mathrm{D}$ geometry of the facades from the vertical laser scans and texture map them using the camera images. Then, different levels of detail are generated and organized in a scene graph in order to enable interactive rendering.

\footnotetext{
*e-mail: frueh@eecs.berkeley.edu, avz@eecs.berkeley.edu
}

\section{Results}

We have applied the proposed algorithms on a large data set acquired during two measurement drives in Berkeley, with a total length of 24.3 kilometers traversed in 78 minutes. A total of 332575 vertical and horizontal scans, consisting of 85 million scan points, along with 19200 images, are captured during those two drives. Using our automated processing steps, we generate facade models for 12 street blocks in downtown Berkeley as shown Figures 2 and 3. The total acquisition time for this model is only 25 minutes; this is the time that it took to drive the total of 8 kilometers around the blocks under city traffic conditions.

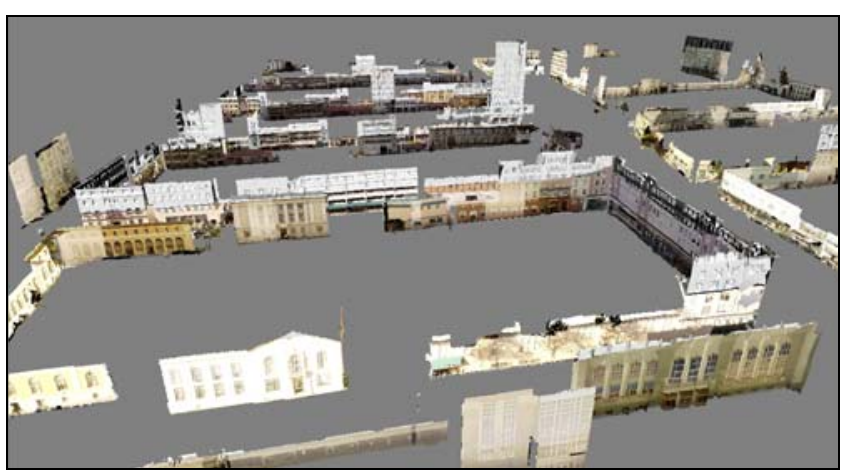

Figure 2. Facade models for downtown Berkeley.
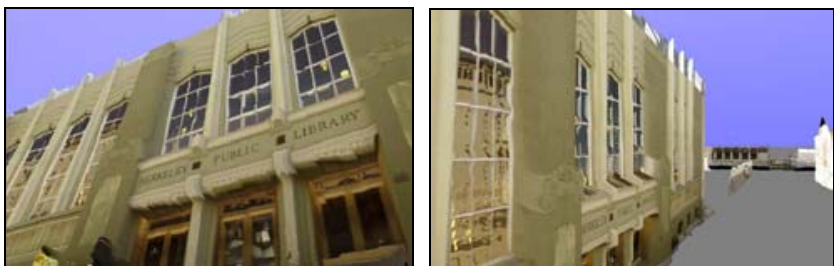

Figure 3. Two close-up views of the Berkeley Public Library.

Our proposed approach to city modeling is not only automated, but also fast from a computational viewpoint: For the twelve downtown blocks shown, the total time for the fully automated processing from the raw data to the final model is 4 hours and 45 minutes on a $2 \mathrm{GHz}$ Pentium-4 PC. Since the complexity of all developed algorithms is linear in path length, our method is scalable and applicable to large environments.

\section{References}

FRUEH, C., AND ZAKHOR, A. 2001(a). Fast 3D model generation in urban environments. In Multisensor Fusion and Integration for Intelligent Systems, Baden-Baden, Germany, p. 165-170

FRUEH, C., AND ZAKHOR, A. 2001(b). 3D model generation of cities using aerial photographs and ground level laser scans. In Computer Vision and Pattern Recognition, Hawaii, USA, p. II-31-8, vol.2. 2

FrueH, C., AND ZAKHOR, A. 2002. Data Processing Algorithms for Generating Textured 3D Building Facade Meshes From Laser Scans and Camera Images. In $3 D$ Processing, Visualization and Transmission 2002, Padua, Italy, p. $834-847$ 